\title{
Redes de cooperación interuniversitaria Canarias - África: investigación, innovación y evaluación desde metodologías colaborativas en políticas de igualdad
}

\author{
González Pérez, María Inmaculada \\ Universidad de La Laguna, España \\ migonpe@ull.edu.es \\ Ascanio Sánchez, Carmen \\ Universidad de La Laguna, España \\ cascanio@ull.edu.es \\ García Cuesta, Sara \\ Universidad de La Laguna, España \\ sagarcia@ull.edu.es
}

\section{Resumen}

La presente comunicación pretende dar a conocer el proyecto de investigación que se iniciará en el año 2018 gracias a la financiación de Gobierno Autónomo de Canarias, teniendo como objetivo general contribuir al posicionamiento de la cooperación canaria como referente en materia de políticas de igualdad de género en el contexto atlántico-africano.

Esto se llevará a cabo mediante la conformación de una Red entre universidades canarias y las de países de África del Oeste centrada en el diseño, aplicación y evaluación de políticas de igualdad, a través del desarrollo de metodologías colaborativas innovadoras en contextos universitarios. Las universidades participantes son la Universidad de La Laguna (España), la Universidad de Las Palmas de Gran Canaria (España), la Universidad de Cabo Verde (Cabo Verde), la Universidad de Letras y Ciencias Humanas de Bamako (Mali), la Universidad de Nuakchot (Mauritania) y la Universidad Hassan II (Marruecos).

A través de esta red se compartirán conocimientos, experiencias y propuestas en el diseño innovador de políticas de igualdad, con especial atención a la diversidad cultural y las propuestas endógenas. Para ello se requiere realizar diagnósticos compartidos, resultado de los procesos colaborativos, desarrollar materiales para la divulgación conjunta y transferencia, así como formar personal especializado en las cuestiones implicadas. Relacionado con lo anterior, se llevarán a cabo tanto acciones comunes y compartidas para alcanzar las metas propuestas en los diagnósticos, como otras específicas y adaptadas a las necesidades de cada institución y país. De manera transversal, constituyendo un principio que rige todo el proyecto, se fortalecerá el papel de las universidades en cada territorio en su compromiso con las políticas públicas y la transferencia al tejido productivo de sus entornos.

\section{Abstract}

The present communication aims to publicize the research project that will begin in 2018 thanks to the financing of the Autonomous Government of the Canary Islands. The general objective is to contribute to the positioning of the Canarian cooperation as a reference in terms of policies of gender equality in the Atlantic-African context. This will be carried out through the creation of a Network between Canarian universities and those of West African countries focused on the design, application and evaluation of equality policies, through the development of innovative collaborative methodologies in university contexts. The participating universities are the University of La Laguna (Spain), the University of Las Palmas de Gran Canaria (Spain), the University of Cape Verde (Cape Verde), the University of Letters and Human Sciences of Bamako (Mali), the University of Nouakchott (Mauritania) and the University Hassan II (Morocco).

Through this network, knowledge, experiences and proposals will be shared in the innovative design of equality policies, with special attention to cultural diversity and endogenous proposals. For this purpose, it is necessary to carry out shared diagnostics, which would be the result of collaborative processes, develop materials for joint dissemination and transfer, as well as train specialized personnel in the issues involved. Related to the above, common and shared actions will be carried out to reach the goals proposed in the diagnoses, as well as specific ones adapted to the needs of each institution and country. In a transversal manner, constituting a principle that governs the entire project, the role of universities in each territory will be strengthened in their commitment to public policies and the transfer to the productive fabric of their environments.

Palabras clave: Género, Educación Superior, África Occidental, Cooperación Educativa Internacional, Inclusión.

Keywords: Gender, Higher Education, West Africa, International Cooperation in Education, Inclusion. 
El proyecto que se presenta pretende conformar una Red entre universidades canarias y las de países de África del Oeste centrada en el diseño, aplicación y evaluación de políticas de igualdad, a través del desarrollo de metodologías colaborativas innovadoras en contextos universitarios. En principio, las universidades participantes son la Universidad de La Laguna (España), la Universidad de Las Palmas de Gran Canaria (España), la Universidad de Cabo Verde (Cabo Verde), la Universidad de Letras y Ciencias Humanas de Bamako (Mali), la Universidad de Nuakchot (Mauritania) y la Universidad Hassan II (Marruecos). El equipo responsable del proyecto trabaja en la Universidad de La Laguna.

Para ello se propone, en primer lugar, crear y desarrollar una red de cooperación mediante procesos colaborativos, con el objetivo de compartir conocimientos, experiencias y propuestas en el diseño innovador de políticas de igualdad, con especial atención a la diversidad cultural y las propuestas endógenas. A lo largo del proyecto se realizarán diagnósticos compartidos, resultado de los procesos colaborativos, desarrollaran materiales para la divulgación conjunta y transferencia y se formará personal especializado en las cuestiones implicadas. Relacionado con lo anterior, se llevarán a cabo tanto acciones comunes y compartidas para alcanzar las metas propuestas en los diagnósticos, así como otras específicas y adaptadas a las necesidades de cada institución y país.

De manera transversal, constituyendo un principio que rige todo el proyecto, se pretende fortalecer el papel de las universidades en cada territorio en su compromiso con las políticas públicas y la transferencia al tejido productivo de sus entornos.

Es importante destacar que las universidades socias se han comprometido a fomentar en sus estructuras y funcionamiento el principio de no discriminación por razón de género y la igualdad sustantiva o real, tal como vienen definidos en los instrumentos del sistema de Derechos Humanos de Naciones Unidas.

El desarrollo del propio proyecto se basa en dos pilares éticos fundamentales, que comparten las universidades socias y que le dan coherencia al proyecto:

1. Presencia equilibrada de hombres y mujeres en todos los espacios de toma de decisiones como requisito democrático básico para la construcción de la igualdad.

2. Participación de la comunidad universitaria en línea con las metodologías participativas y de colaboración propuestas en el diseño de este proyecto.

Este proyecto de I+D+ I está financiado por la Agencia Canaria de Investigación, Innovación y Sociedad de la Información de la Consejería de Economía, Industria, Comercio y Conocimiento del Gobierno de Canarias. Y cuenta con el apoyo de la Fundación General de la Universidad de La Laguna y el Excelentísimo Cabido Insular de Tenerife.

\section{OBJETIVOS ESPECÍFICOS Y RESULTADOS}

- Objetivo 1: Desarrollar y consolidad redes de cooperación para la puesta en marcha y adaptación a cada contexto de políticas de igualdad y, por tanto, la internacionalización de las universidades.

- Resultado 1.1. Acuerdos de compromiso de todos los miembros para conformar la red.

- Resultado 1.2. Diseño de una página web para el trabajo cooperativo en red (Group Ware).

- Objetivo 2: Diseñar y aplicar un proceso colaborativo a través de metodologías innovadoras que permitan el intercambio de conocimientos y experiencias multinivel.

- Resultado 2.1. Informes autodiagnósticos.

- Resultado 2.2. Informe de diagnóstico colaborativo.

— Resultado 2.3. Jornadas de presentación y divulgación de los diagnósticos. 
- Resultado 2.4. Diseño y aprobación conjunta del sistema de indicadores de evaluación.

— Resultado 2.5. Dos publicaciones del diagnóstico común.

- Objetivo 3: Identificar las distintas realidades y procesos que acompañan en cada contexto territorial el desarrollo de las políticas de igualdad, con especial atención a la diversidad y a las propuestas endógenas.

- Resultado 3.1. Producciones de tipo metodológico compartidas y como resultado del proceso colaborativo:

- Resultado 3.2. Manual de uso con las propuestas metodológicas innovadoras para el diseño, la implantación y evaluación de políticas de igualdad en las instituciones universitarias.

- Resultado 3.3. Producción de documental-audiovisual. Colaboración de ULL Media y producción (concurso de ideas y producción) con posible colaboración de estudiantado de diseño de la Universidad de La Laguna y de la ULPGC.

- Resultado 3.4. Formación en políticas de igualdad en contextos universitarios en las jornadas de reuniones en la Universidad de La Laguna.

- Objetivo 4: Diseñar medidas producto de los diagnósticos y trabajo procesual previo realizado, que puedan ser, tanto comunes y compartidas, como específicas y adaptadas a las necesidades de cada institución/país.

- Resultado 4.1. Planes, protocolos, guías y otras medidas concretas para igualdad de oportunidades, comprometidas y firmadas en los convenios de compromiso.

- Resultado 4.2. Informe-diagnóstico de evaluación, exponiendo las potencialidades para el futuro de la red.

- Resultado 4.3. Jornadas Internacionales para dar a conocer el proceso y las acciones desarrolladas a lo largo del proyecto, tanto en el contexto universitario como en el resto de la sociedad.

- Objetivo 5: De modo transversal, se pretende fortalecer el papel de las universidades en cada territorio desde su compromiso con las políticas públicas, la gobernabilidad, la cooperación y la transferencia al tejido productivo.

- Resultado 5.1. Desarrollo de planes de igualdad u otro tipo de políticas de igualdad en las universidades implicadas.

- Resultado 5.2. Fomento de las relaciones institucionales formalizadas en tipos de convenios.

- Resultado 5.3. Formación de agentes de igualdad en cada institución.

- Resultado 5.4. Transferencia al sector productivo de resultados producidos y de profesionales formados a través de la red. Por ejemplo, aplicación de políticas de igualdad en el sector empresarial, propuesta de desarrollo normativo, formación de formadores, incorporación de criterios con perspectiva de género en la investigación, diseño de indicadores adaptados a cada contexto que visibilice las desigualdades y los avances conseguidos.

\section{METODOLOGÍA: SUS FASES}

La metodología colaborativa que será utilizada en el proyecto se traduce en un marco de desarrollo procesual de trabajo conjunto de todas las personas y entidades participantes, hacia la formación y consolidación de una Red que se comprometa a trabajar en las medidas y producciones comunes y específicas asociadas a esta. 


\section{A. Primera Fase}

El primer paso es la propia configuración de la Red, con los primeros objetivos formales centrados en un autodiagnóstico de cada institución participante en los términos indicados por los objetivos: los diferentes niveles de posicionamiento de cada institución/país en referencia a la situación de hombres y mujeres en sus universidades, las aplicaciones normativas, las políticas de igualdad y la gobernabilidad (diagnósticos parciales).

Estos diagnósticos se presentarán en una primera reunión general (2018) del proyecto. Este encuentro se iniciará con un simposio de una puesta en común. Posteriormente se desarrollarán talleres específicos en los que se plantean puntos comunes y diversidades, para establecer acuerdos de posible compromiso de cada equipo e iniciar el intercambio de instrumentos. El trabajo realizado en los talleres permitirá definir las líneas de necesidades conjuntas, junto con propuestas y asesoramientos conjuntos sobre problemas específicos que solo afecten a uno de los/as socios/as.

En esta misma reunión tendrá lugar la presentación de una web que tendrá como objetivo la divulgación del proyecto así como posibilitar el trabajo conjunto que facilitará a su vez la siguiente meta del proceso: el diagnóstico colectivo.

\section{B. Segunda Fase}

Este diagnóstico se desarrolla en los seis meses siguientes, con un volcado de información a través de los medios telemáticos (página web, reuniones en streaming, etc.) que permitirá general un documento común para plasmar:

a. la diversidad de situaciones y niveles de desarrollo, implementación y evaluación de las políticas de igualdad en cada institución.

b. Ios puntos comunes de desarrollo, expectativas y acuerdos conjuntos para trabajar a través del diseño de medidas e indicadores comunes y/o adaptados a todas las entidades participantes.

c. el diseño del sistema de evaluación negociado y procesual que sirva para concretar los compromisos y metas de cada institución/país en un escenario común.

Al final de este período será generado y divulgado el primer diagnóstico colectivo, en el que se ofrecerán además las herramientas para la posterior implementación de las acciones: protocolos éticos de diseño, implementación y evaluación; listado de posibles ejes y medidas de actuación; sistemas de evaluación (indicadores cuantitativos/cualitativos), formas concretas de aplicación de mejoras y transferencias sociales asociadas a las propuestas. Una Evaluación Interperíodo (2018) por parte del grupo motor y bajo las premisas alcanzadas por la Red previamente, permitirá realizar un seguimiento del proceso, junto con las posibilidades que permite la página web, en el marco de las circunstancias de cada institución/país.

\section{Tercera Fase}

La siguiente fase del Plan se inicia con la Aprobación formal de las herramientas que cada institución haya decidido elaborar, bajo las premisas señaladas por el diagnóstico colectivo y los acuerdos y protocolos de actuación firmados. La aprobación será formulada en términos de Planes, Protocolos, Guías y otras medidas a aplicar en cronograma, en cada entorno. Estas herramientas serán supervisadas por el grupo motor y compartidas on-line para el debate de toda la Red. El seguimiento en este proceso se realizará mediante una Evaluación Interperíodo (2019). Ésta será llevada a cabo por el grupo motor atendiendo las premisas previamente establecidas por la Red. Para ello se explotarán todas las posibilidades que permite la página web, en el marco de las circunstancias de cada institución/país. 


\section{Cuarta Fase}

La implementación de las medidas diseñadas para cada institución tendrá lugar en los siguientes meses. Para el correcto funcionamiento metodológico, este proyecto requiere un servicio muy activo de traducción disponible para los encuentros colectivos, y también para el intercambio viable de información, herramientas y resultados on-line. A su vez, serán necesarios los servicios telemáticos ULL y otros (tales como alquiler de equipos audiovisuales, salas especiales, servicios de traducción).

A su vez, para poder alcanzar los resultados previstos en el proyecto, los miembros del equipo requerirán de asesoramiento de personas expertas en metodologías colaborativas y en políticas de igualdad en instituciones públicas; así como de apoyo técnico y administrativo en momentos clave.

Esta última Fase concluirá con una Evaluación Final (2020) atendiendo a los criterios acordados por todas las entidades participantes en el proyecto.

\section{EVALUACIÓN DEL PROYECTO}

En el proyecto se contemplan tres evaluaciones: dos interperiódicas y una final (ya indicadas anteriormente). Se plantean estas evaluaciones a través de diferentes aplicaciones técnicas. Una parte de ellas será realizada a través de la Red, en consonancia con el tipo de indicadores que parezcan más adecuados a las posibilidades.

A su vez, se plantea un Análisis Delphi a un grupo de expertas/os, con miembros de todas las entidades comprometidas. Las tres oleadas del Delphi -una en cada año desde el 2018 al 2020- permitirán una reflexión informada sobre el proceso en sus períodos incipiente, intermedio y final y facilitará la apertura de la Red a otros miembros de las propias instituciones y otros organismos de conocimiento científico del país. El grupo motor realizará un seguimiento del Delphi, cuyo soporte de recogida de datos será el adecuado a las posibilidades que proponga la Red en su primer encuentro.

\section{CONCLUSIONES}

Difícilmente pueden establecerse conclusiones respecto a un proceso que acaba de iniciar sus pasos, pero sí pueden avanzarse las actividades que se encuentran en marcha en estos momentos:

Gráfica 1: Tareas iniciales red CIMPI.

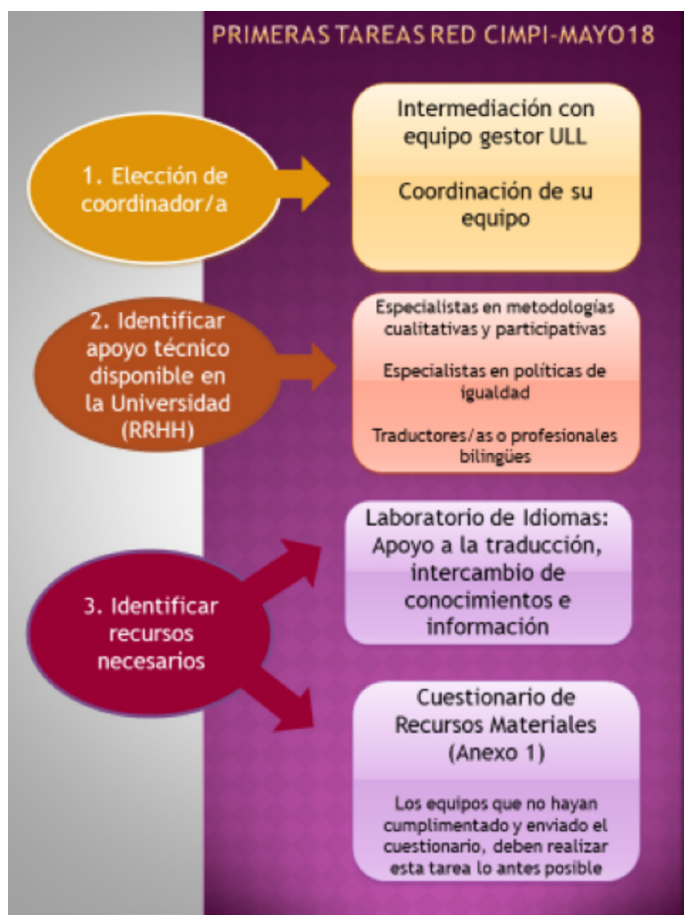


Además de estas actividades principales, se está procediendo a la redacción y firma de los necesarios convenios generales y específicos de colaboración con las universidades implicadas.

Se están recopilando indicadores de medición internacionalmente reconocidos en materia de igualdad de género para su análisis y selección en la primera reunión general que tendrá lugar el noviembre de 2018.

Y se está elaborando la página web con el apoyo de técnicos informáticos con el objeto de poner las TIC al servicio de los procesos de investigación, innovación y evaluación desde metodologías colaborativas en políticas de igualdad.

\section{REFERENCIAS BIBLIOGRÁFICAS}

Assié-Lumumba, N. (coord.) (2009). Mujeres en África: educación y poder. El acceso a los estudios superiores. Madrid: IEPALA. Tomo I, pp. 92-126. Brugué, Quim. ¿Qué estrategias para qué retos? 25 criterios para el diseño de estrategias en el desarrollo local. Diputación de Barcelona, 2014.

EURI-CRUE. Estrategia de cooperación universitaria al desarrollo, 3 de marzo de 2000. Córdoba. CRUE. 2000.

Khelfaoui, Hocine, Introduction. Le Processus de Bologne en Afrique: globalisation ou retour à la " situation coloniale»? JHEA/RESA Vol. 7, Nos. 1\&2, pp. 1-20, Conseil pour le développement de la recherche en sciences sociales en Afrique, 2009.

Makosso, Bethuel, La crise de l'enseignement supérieur en Afrique francophone: une analyse pour les cas du Burkina Faso, du Cameroun, du Congo, et de la Côte d'Ivoire. En JHEA/RESA Vol. 4, No. 1, pp. 69-86, Conseil pour le développement de la recherche en sciences sociales en Afrique, 2006.

Obasi, Isaac N. y Olutayo, Akinpelu O. Globalization, the Bologna Processand African Universities: Limitsand Contradictions of Market-orientedHigher Education Reforms. Council for the Development of Social Science Research in Africa 2009. JHEA/RESA Vol. 7, Nos. 1 y 2, pp. 159-187, Conseil pour le développement de la recherche en sciences sociales en Afrique, 2009.

Palma, Montserrat. Marco legislativo actual de la transferencia de conocimiento en España; en Testar, Xavier. La transferencia de tecnología y conocimiento universidad-empresa en España: estado actual, retos y oportunidad. Colección Documento CYD. Barcelona. Universitat de Barcelona. 2012. Pp. 9-12.

Regulation (EU) $n^{\circ} 1291 / 2013$ of the European Parliament and of the council of 11 December 2013 establishing Horizon 2020 - the Framework Programme for Research and Innovation (2014-2020) and repealing Decision No 1982/2006/EC.

UNESCO (1998): Declaración Mundial sobre la Educación Superior en el siglo XXI: Visión y acción; en www.unesco.org/ education/educprog/wche/declaration_spa.htm, consultado el 1 de marzo de 2017. 